\title{
Structural Design on a New Type Magnetorheological Fluid Retarder
}

\author{
Wang Yuexin ${ }^{1, a}$, Zhang Guosheng ${ }^{2, b}$ Huang Shenshui ${ }^{3, c}$ \\ ${ }^{1}$ Longyan University Mechanical and Electronic Engineering Institute Longyan Fujian China \\ ${ }^{2}$ Research Institute of Highway Ministry of Transport Beijing China \\ ${ }^{3}$ Longyan University Mechanical and Electronic Engineering Institute Longyan Fujian China \\ awyx11111@163.com, b990165733@qq.com, ㄷ4892542@qq.com
}

\begin{abstract}
Keywords: Magnetorheological fluid, Retarder, Configuration, Design
Abstract. Magnetorheological fluid (MRF) is a kind of rheological property which can produce fast changing material under the action of magnetic field. The rheological properties of MRF can be changed by controlling the strength of magnetic field. When there is no magnetic field, its characteristics appear as Newton fluid characteristics. When an external magnetic field is applied, it exhibits the behavior of Binghan plastic body with cohesive and plastic properties. Recent years, out of vehicle safety consideration, retarder is more and more widely used, this paper adopted MRF in vehicle retarder, together with water pump concept, a new type of configuration of retarder is designed. This retarder has the following advantages: short response time, easy control, low energy consumption and simple structure.
\end{abstract}

\section{Introduction}

Heavy duty truck need supplement braking system, supplement braking system is not a configuration to make the automobile stop, but a system makes automobile slow down or move stably. especially move down a long slope road, so retarder are used in such vehicles, but recent retarder are all hydraulic system, heavy, complex configuration. MRF nowadays widely used in damper to absorb the vibration energy, why MRF cannot be used in retarder to replace normal hydraulic fluid, this is our research's target. Utilizing MRF characteristics strengthen the deceleration effect. Magnetorheological fluid mainly include base liquid, magnetic particles and additive(Fig1). No magnetic field, magnetic particles in the base fluid is diffuse irregularity; under the magnetic field, the magnetic particles in the base fluid will concentrate into chain, we call it chain effect, this is the core of magnetorheological effect. The key fact to produce magnetorheological fluid is magnetic particles, the main methods to produce magnetic particles include: crushing method, deposition method, Coping method, soft chemical method and thermal decomposition method and so on.

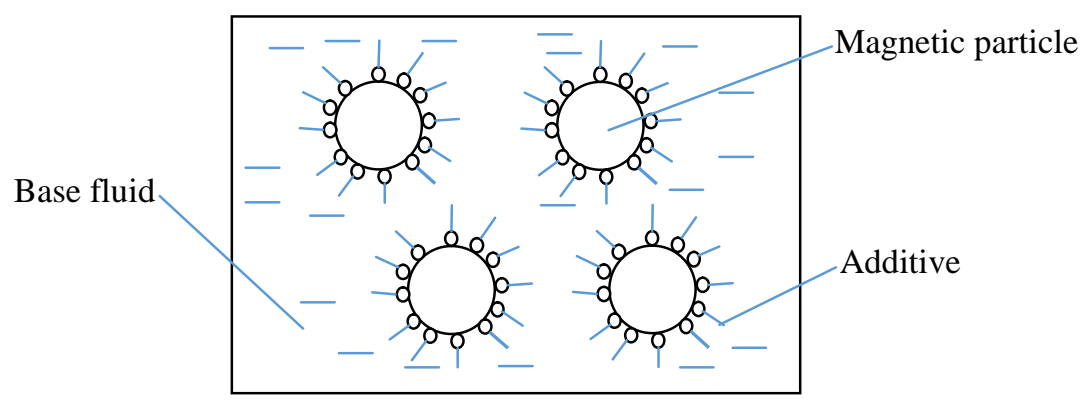

Fig. 1 Content of MRF

When exerting magnetic field, the micro particles in the MRF will move orderly, such movement will begin when the electromagnetic coil is energized, till such orderly movement stop and turn into relatively stability condition, become a fixed structure. It means that these particles attact each other, forming chain structure along the magnetic field direction, this period is called chaining process. Refer with Fig2, under zero magnetic field, micro particles roaming distribution in the base fluid, under this condition dynamic yield stress does not exist; under magnetic field, the micro particles 
form a chain style, viscosity of MRF increase with direction. The number of chains increase, gather together to form a large column, make the viscosity and dynamic yield stress keep on strengthening. External shear stress increases. When the magnetic field is deleted, MRF will restored to their original, re-become the original irregular distribution state, the totally chaining process is only several milliseconds. The magnetic direction will also decide the particles' arrangement direction.
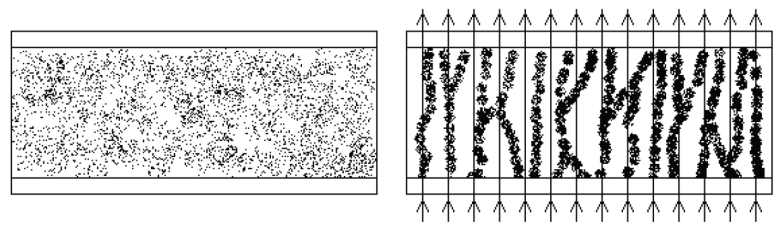

Fig. 2 Magnetic fluids viscosity change. Left - MRF without external magnetic field. Right - Magnetic particles arrangement under magnetic field

\section{Design on MRF retarder}

Initially thinking of putting the brake disk into a container full of MRF, through the electromagnetic coil making MRF viscosity, result in vehicles slowing down, simple and rapid. Further consideration is utilizing pump theory, the impeller of the pump can absorb and drain the water, this can make the MRF cycle in the decelerating chamber, this not only can make MRF heat dissipation but also can make magnetic particles dispersion flow into MRF. When vehicle normal moves, there are no fluid in the decelerating chamber, this cannot produce extra energy consumption, so a tank is designed, when retarder works, need MRF, pneumatic pump push MRF into chamber, power ups to decelerate. When vehicles come into normal driving condition, pneumatic pump pumping, if the pressure in the tanker is lower than the chamber, the MRF will flow back into the tanker, solenoid close, there will be no fluid in the chamber, the impeller resistance force will decrease a lot, engine's energy will get better utilization.

\section{MRF retarder operating principle and configuration}

MRF operating principle is completely different with eddy current and hydraulic retarder, its relative theory include MRF composition, Magnetorheological effect, MRF performance requirements. MRF is the specific non-gel suspension liquid which dispersed micro sized magnetic polarized particles in insulating liquid, so rheological properties will change with magnetic field variation. Without magnetic field, Rheological properties of the MRF is similar with Ordinary Newtonian fluid, under medium intensity magnetic field, Its apparent viscosity coefficient increased by more than two orders of magnitude. Under Strong magnetic field, MRF will turn into solid state, Liquidity disappears; Once the magnetic field is removed, it will turn into flowing liquid again, This reversible transformation can be done in milliseconds. So after power on, the magnetorheological fluid will quickly become viscous, impeller's shear force increased rapidly, making the speed of the impeller slow down, the vehicle speed will decelerate. When vehicles need deceleration, The air pump will enter the air pressure in the A hole, the MRF in the container will flow into decelerating chamber, when the retarder chamber is full of MRF, level sensor response, electronically controlled valve closed. MRF can't flow back into the MRF tank, pneumatic pump shutdown. At the same time while the electromagnetic coil power, resulting in magnetic field, the slow flow in the chamber of the magnetorheological fluid by the magnetic field will become viscous, so as to prevent the rotation of the impeller, play a deceleration effect. When the car is on the gentle road, need not deceleration, the electronic control valve open, the air pump will take out the gas in the magnetorheological tank, magnetorheological tank pressure will be reduced, so that the magnetorheological fluid will flow back into magnetorheological tank, when the top of the tank level sensor response, the electronic control valve closed, so that the magnetorheological fluid will not flow into the decelerating chamber, air pump shut down. There is not MRF in the decelerating chamber, the impeller will not subject to strong resistance, can reduce the engine power loss. 
MRF retarder is consist of mechanical equipment and MRF. MRF retarder's main configuration include retarder cover, MRF tank, deceleration chamber. specific structure is shown in Fig3, include hole A, used to pumping and inhale. Electronically controlled valve is used to decide the retarder working time, and electromagnetic coil which is used to generate magnetic field. Other components like impeller will be introduced in the below paragraph.

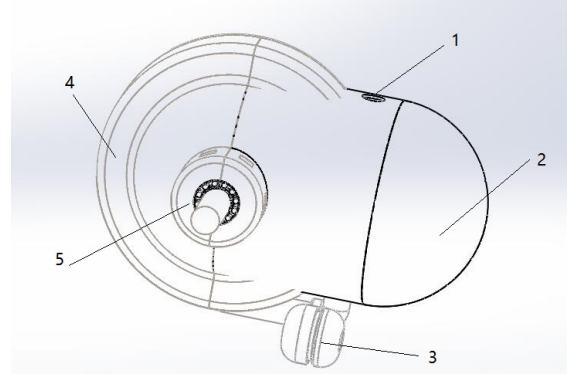

Fig. 3 Retarder illustration

1. Hole A: pump booster / suction, 2. MRF tank, 3. Electronically controlled valve 4,5. Electromagnetic coil installation position

\section{Impeller}

Impeller is one of the key component of this equipment, its size is shown in fig 4, unit is mm. Fig5 show its $3 \mathrm{D}$ diagram. It is connected with the vehicle driving axle, the main function of impeller is absorbing and exhausting the magnetorheological fluid cyclically, under the effect of magnetic field induced by electromagnetic coils, gradually sticky magnetorheological fluid will make the impeller deceleration.

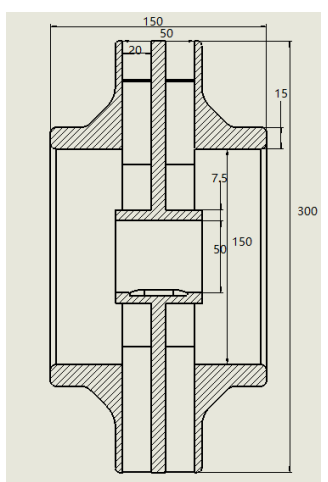

Fig. 4 Impeller size chart

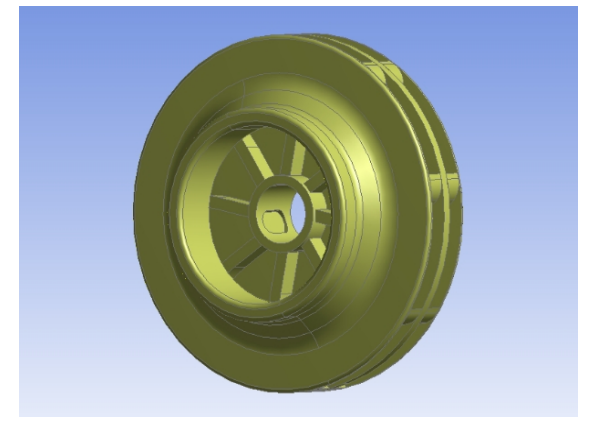

Fig. 5 Impeller diagram

\section{Configuration of MRF retarder}

Fig6 show the size of the retarder, unit is mm, and Fig7 show the 3D diagram of the MRF retarder. The magnetorheological fluid retarder designed in this paper is a device that uses the rheological characteristics of MRF to slow down the vehicle speed. Through the rheological properties of MRF, when the retarder is powered, the MRF changes with the fluid characteristics through the magnetic field. If the magnetic field is stronger, the deceleration effect of MRF will be more obvious. Retarder chamber's effective capacity is $1991 \mathrm{ml}$, water tank's capacity is $2402 \mathrm{ml}$, MRF volume is $2000 \mathrm{ml}$, to keep the tank pressure, the minimum pressure of withdraw is $P=\rho g h=3000 \cdot 9.8 \cdot 0.29=8526^{\mathrm{Pa}}$, think about the several thousands revolutions of the impeller per minute, stress also need to be raised a certain gradient, considering MRF Yield strength is generally $15 \mathrm{kPa}$, this pressure at least reach 10 $\mathrm{kPa}$. 

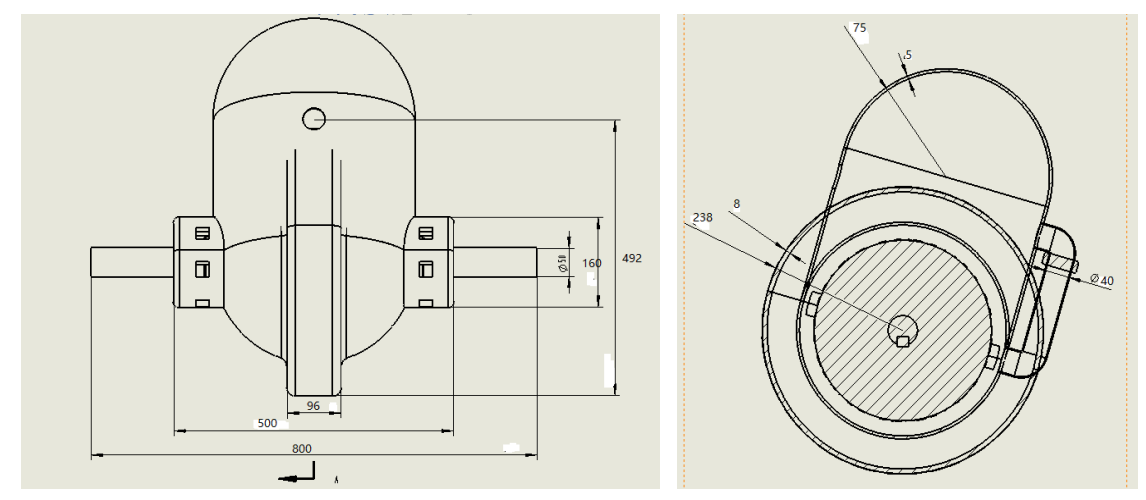

Fig. 6 Size diagram of MRF retarder
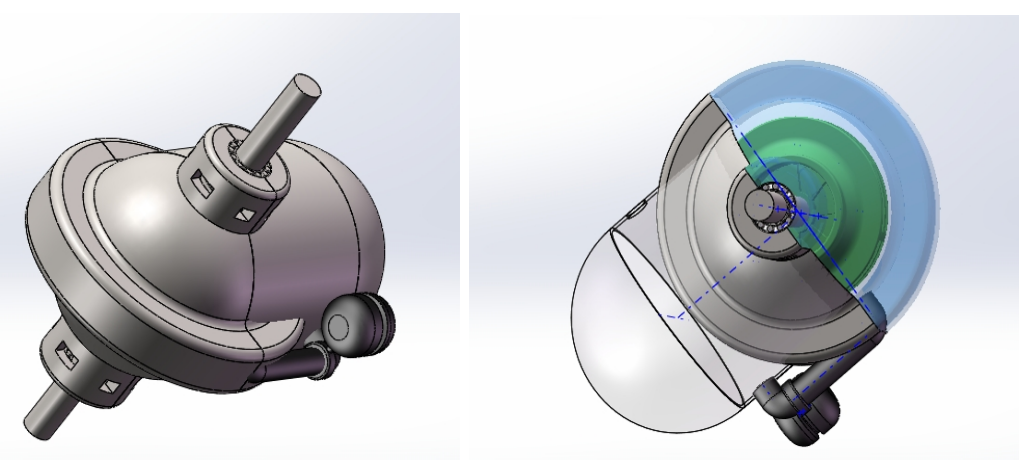

Fig. 7 Totally appearance

\section{MRF cycling in the decelerating chamber}

MRF will cycle in the retarder chamber under impeller's rotating, avoid the magnetic particles contacting tightly against the retarder chamber's inner wall under the high speed rotating's centrifugal force, cycling flow also can decrease MRF's temperature. MRF's cycle in the retarder chamber mainly caused by impeller's high speed rotating on the driving axle, the MRF within the impeller will be thrown to the outside of the impeller, the MRF on the both ends of the retarder chamber will be added to the impeller, do such cycle action. When the vehicle move into the gentle zone, MRF will be withdrawn to the tank. The impeller connect with the axle through splined hub, MRF will quickly become sticky by the role of external magnetic field, the shearing force required for the impeller increases, So the impeller and its associated drive shaft will be significantly slow down.

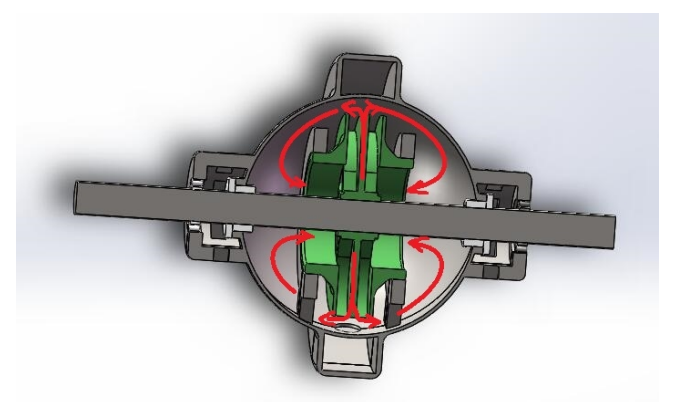

Fig. 8 Flow direction of MRF 


\section{Conclusions}

This paper starts with the introduction of MRF, analyzing the deceleration effect of MRF retarder and the advantages of the magnetorheological fluid retarder compared to the eddy current retarder and hydraulic retarder. The concept of the pump is introduced into the design of the magnetorheological fluid retarder, the overall structure design is carried out, get the initial MRF retarder structure. The MRF retarder has the characteristics of light weight and easy operation. MRF is new technology emerging in recent years, we put our new design idea in retarder design, concrete application needs further simulation experiment argument.

\section{Acknowledgements}

This work was financially supported by the Fujian Natural Science Foundation (2017J01706).

\section{References}

[1] David Case, Behazad Taheri and Edmond Richer. Design and Characterization of a Small-Scale Magnetorheological Damper for Tremor Suppression, Transactions on Mechatronics, Vol. 18 (2013),p.96-103

[2] Muhammad Mahbubur Rashid, Nasrudin A. Rahim. Analysis and Experimental Study of Magnetorheological-Based Damper for Semiactive Suspension System Using Fuzzy Hybrids. IEEE Transactions on Industry Applications, Vol. 47(2011),p.1051-1059

[3] Jianbin Zeng, Youguang Guo, Jianguo Zhu. Investigation and Simulation on Magnetic Hysteresis

Properties of Magnetoheological Fluid. IEEE Transactions on Industrial Electronics. Vol. 64(2017),p.1611-1616

[4] Jonathan M. Chambers, Norman M. WereleyVertical Axis Inductance Monitoring System to Measure Stratification in a Column of Magnetorheological Fluid. IEEE Transactions on Mechatronics, Vol. 53 (2017)

[5] A. Musolino, M. Raugi, R. Rizzo and E. Tripodi. A Passive Clutch based on MagnetoRheological Fluids and Eddy Currents. 2016 IEEE $2^{\text {nd }}$ International Forum on Research and Technologies for Society and Industry Leveraging a better tomorrow(RTSI). 978

[6] Banna Kasemi, Asan G.A. Muthalif, M.M. Rashid, Mahmudur Rahman. Optimizing Dynamic Range of Magnetorheological Fluid Dampers: Modeling and Simulation. 2011 4th International Conference on Mechatronics (ICOM)

[7] Manuel A. Fernández, Jen-Yuan (James) Chang. Development of Magnetorheological Fluid Clutch for Robotic Arm Applications. IEEE Advanced Motion Control 